\section{A organização da Atenção Básica em municípios integrantes do Projeto de Expansão e Consolidação do Saúde da Família em Mato Grosso, Brasil}

\author{
Organization of Primary Health Care in cities \\ belonging to Project for Expansion and \\ Consolidation of the Family Health Strategy in \\ Mato Grosso State, Brazil
}

Elza Machado de Melo 1,2

Lúcia Paiva 2,3

Juliana Álvares 2

André Luiz Dumont Flecha ${ }^{2}$

\author{
${ }^{1}$ Faculdade de Medicina, \\ Universidade Federal de \\ Minas Gerais, \\ Belo Horizonte, Brasil. \\ 2 Núcleo de Estudo em Saúde \\ Coletiva, Universidade \\ Federal de Minas Gerais, \\ Belo Horizonte, Brasil. \\ 3 Secretaria Municipal de \\ Saúde de Belo Horizonte, \\ Belo Horizonte, Brasil. \\ Correspondência \\ E. M. Melo \\ Departamento de Medicina \\ Preventiva, Faculdade de \\ Medicina, Universidade \\ Federal de Minas Gerais. \\ Av. Alfredo Balena 190, \\ Belo Horizonte, $M G$ \\ 30130-100, Brasil. \\ elzamelo@medicina.ufmg.br
}

\begin{abstract}
This article presents part of the results from the Baseline Study on the PROESF. The objective was to evaluate primary health care in the cities of Cuiabá, Várzea Grande, and Rondonópolis, Mato Grosso State, Brazil, based on the inter-subjectivity in human relations (among health workers, users of health services, and the public at large and within institutionalized levels of social control). A qualitative and quantitative methodology was used, including interviews with key informants; short meetings with managers; focal groups with managers; and interviews with users and health professionals from pre-selected health units. Scores were assigned to all the questions that indicated participatory processes in primary care practices in the various municipalities. Despite the geopolitical identity among the municipalities and their similar access to the same public policies, there was a significant difference in their performance of the functions pertaining to the organization of primary care and the Family Health Program, in terms of portal of entry into the system, longitudinality, comprehensiveness, and coordination. Differences were observed in the type of relations that were established (participatory versus non-participatory), corresponding to the previous difference.
\end{abstract}

Primary Health Care; Evaluation; Family Health Program
Introdução

A saúde é um bem de valor incontestável e se refere a necessidades legítimas dos seres humanos, definidas por profissionais de saúde ou percebidas pelo próprio indivíduo 1,2 . Fatores biológicos, políticos, sociais, econômicos, ambientais e culturais e os relativos à prestação de cuidados pelo sistema de saúde interferem no processo de produção da saúde, impondo aos profissionais e aos serviços abordagens mais amplas e complexas do que aquelas centradas no cuidado curativo 1,3,4. Soma-se a essa complexidade, outra de ordem tecnológica e, juntas, elas explicam por que a produção de saúde representa um grande desafio para a sociedade e exige o desenvolvimento de novos modelos de atenção: a ênfase à Atenção Básica, dentro de um sistema de saúde regionalizado e hierarquizado, é a principal proposta disponível, representando uma tendência mundial na organização dos serviços de saúde 4,5,6.

O presente trabalho, cujo objetivo é avaliar a atenção básica dos municípios de Cuiabá, Várzea Grande e Rondonópolis no Estado de Mato Grosso, é um dos produtos do Estudo de Linhas de Base do Projeto de Expansão e Consolidação do Saúde da Família (PROESF), de âmbito nacional, proposto pelo Ministério da Saúde com o objetivo de avaliar o estágio atual de organização da atenção básica em municípios brasileiros com mais de 100 mil habitantes, o que servirá como 
parâmetro de comparação para avaliações futuras. Os três municípios estudados no presente trabalho são integrantes de um dos dez lotes do estudo e foram agrupados pela sua identidade geopolítica.

Cuiabá, capital do Estado de Mato Grosso, Rondonópolis e Várzea Grande contam com uma população de 542.861, 169.814 e 254.736 habitantes 7 e com índice de desenvolvimento de 0,821, 0,793 e 0,790 , respectivamente 8 . A estrutura de saúde de Cuiabá compreende 18 hospitais, sendo dois públicos, 15 privados e um universitário. Sua rede de atenção básica é constituída por 29 unidades com 29 equipes do Programa Saúde da Família (PSF), trinta unidades básicas de saúde tradicionais (vinte delas contam com Programa de Agentes Comunitários de Saúde - PACS), cinco policlínicas e sete pólos de assistência rural. Várzea Grande, município contíguo ao de Cuiabá e dele separado por uma ponte, conta com um total de 29 estabelecimentos de saúde, sendo seis hospitais (um público e cinco privados). Sua atenção básica é constituída de nove centros de saúde tradicionais, três unidades com nove equipes de saúde da família, um centro de especialidades, quatro policlínicas, sete equipes do PACS. Rondonópolis dispõe de oito hospitais (um público, dois filantrópicos e cinco privados) e de uma rede básica formada por 25 unidades com 28 equipes do PSF, cinco centros de saúde, uma policlínica, um posto urbano, 14 minipostos da zona rural. O município dispõe de uma farmácia de manipulação que garante suficiência de medicamentos da farmácia básica.

Esta pesquisa foi aprovada pelo Comitê de Ética da Faculdade de Medicina da Universidade Federal de Minas Gerais, teve o consentimento dos gestores e todos os entrevistados assinaram termo de consentimento livre e esclarecido.

\section{Uma distinção necessária}

O conceito de Atenção Básica adotado toma por referência o conceito de Atenção Primária, talhado em Alma-Ata, isto é, "atenção essencial à saúde baseada em tecnologia e métodos práticos, cientificamente comprovados e socialmente aceitáveis, tornados universalmente acessíveis a indivíduos e famílias na comunidade por meios aceitáveis para eles..." 9 (p. 16).

A Atenção Primária, muitas vezes, é confundida com "medicina dos pobres" - cuidados simplificados e de baixo custo - sendo objeto de críticas de muitos autores 5,6,10,11, porque configura um tipo de compensação social destinada a mascarar desigualdades sociais, aliviar tensões e garantir a "fidelidade das massas", além de ser garantia de ampliação do mercado para populações que não dispõem de poder aquisitivo.

Sendo assim, a Atenção Primária como um modelo conseqüente de atenção à saúde $4,5,9$ deve ser diferenciada de modelos que representam mero transporte da prática de consultórios médicos para as unidades de saúde e de modelos exclusivamente poupadores de recursos e/ou geradores de controle e dominação. Tomando por fundamento a Teoria da Ação Comunicativa de Habermas, postulamos que só é possível garantir essa distinção se o cuidado de saúde for fruto do exercício da autonomia dos atores sociais envolvidos, condição em que, nas palavras de Habermas 12,13 para designar o conceito de autonomia, são autores dos direitos diante dos quais são destinatários.

O conceito de autonomia, engendrado com base na prática comunicativa cotidiana, é definido como instituticionalização dos processos discursivos de formação da opinião e da vontade, resultante da interpenetração entre princípio do discurso e direito, num processo circular em que o primeiro confere legitimidade ao segundo e este, por sua vez, institucionaliza o primeiro, gerando, ao mesmo tempo, direito legítimo e princípio da democracia, que são, portanto, co-originários 12,13,14 (Figura 1). Aqui já se evidencia o pressuposto deste trabalho: não há direito legítimo que não se vincule à democracia.

Figura 1

Gênese do direito e da democracia.

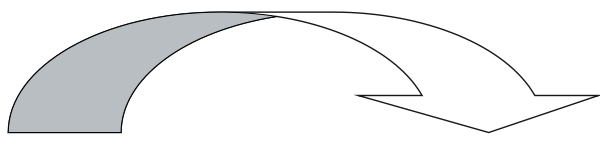

$\begin{array}{ll}\text { Princípio do discurso Direito } & \end{array}$

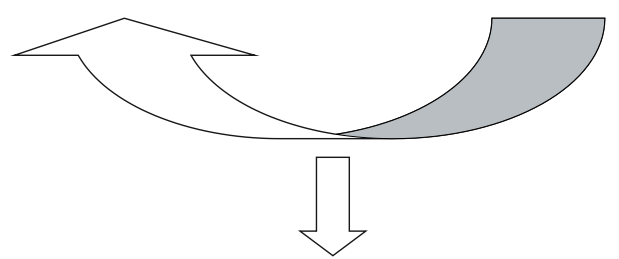

Princípio de democracia Direito legítimo

Criado por E. M. Melo, a partir da Teoria da Ação Comunicativa de Habermas. 
Por impossibilidade de demonstrá-los aqui, os nexos entre discurso e ação comunicativa serão dados como conhecidos. Eles são amplamente discutidos em vários textos de Habermas 12,15,16,17,18 e também em texto da primeira autora ${ }^{14}$. Mais importante para o momento é discutir a transição do modelo de democracia proposto para os processos políticos reais, nas palavras de Habermas, a tensão externa entre validade e facticidade 12,13. Para tanto, Habermas propõe um modelo procedimental de democracia, a política deliberativa, constituída necessariamente por dois complexos, o primeiro, legalmente regulado, que é o sistema representativo e o segundo, a esfera pública, na qual o primeiro se ancora firmemente.

A esfera pública é uma rede comunicacional de informações e opiniões, enraizada na sociedade civil e capaz de detectar, identificar e tematizar os problemas que os atores sociais experimentam, no seu dia-a-dia, frente às externalidades causadas pelos diferentes sistemas. Como toda estrutura do Mundo da Vida, a esfera pública é reproduzida pela ação comunicativa e se refere ao espaço social por ela gerado - "qualquer encontro de atores que se atribuem... reciprocamente liberdades comunicativas, abre-se como espaço público lingüisticamente constituído" 12 (p. 361). Da mais simples interação, esse espaço pode se expandir, incorporando sucessivamente novos atores, generalizando-se, distanciando-se cada vez mais dos contextos específicos, vinculando-se portanto, à cidadania em geral, mas sem perder o eco das experiências individuais. Assim se constitui o debate público, que dramatiza os problemas e necessidades até o ponto em que têm de ser tratados pelas instâncias institucionalizadas do complexo representativo. Em outras palavras, a esfera pública não legisla, mas cria a situação e as antecipações que levam as instâncias representativas a apreciar determinadas matérias, tomar decisões a respeito e aprovar soluções na forma de leis, políticas, programas e/ou projetos.

Até aqui, estivemos falando de mecanismos de integração social que são parte do Mundo da Vida. Mas, Habermas concebe a sociedade como mundo da vida e sistema. O primeiro é o âmbito da ação comunicativa, isto é, toda interação coordenada pelo entendimento lingüístico, processo estabelecido segundo uma relação sujeito-sujeito, mediada pela linguagem no seu uso comunicativo cotidiano. O segundo é o âmbito da ação estratégica, orientada para o êxito, de tal modo que o outro entra no cálculo de cada ator como uma variável, configurando uma relação entre sujeito e objeto 12,15,16. Por conseqüência, o mecanismo de regulação em cada âmbito é diferente, se no mundo da vida faz-se pela in- tegração social, em que os atores sociais harmonizam entre si as orientações das suas ações, no sistema, faz-se por auto-regulação, acima e independente de todos, dada pelo agregado das conseqüências das ações de cada ator social isolado 12,15,19,20,21,22. A integração social se faz por sujeitos que se reconhecem reciprocamente, sendo portanto mediada pela solidariedade. A integração sistêmica é mediada pelo poder e pelo dinheiro, sendo própria do sistema econômico e do sistema administrativo. A saúde, à luz dessa teoria, seria, então, resultante do confronto entre esses elementos - poder, dinheiro e solidariedade - que expressam, respectivamente, os imperativos oriundos do sistema administrativo, do sistema econômico e da opinião e vontade coletiva dos cidadãos, no exercício da sua autonomia 14,23. Com essa abordagem, é possível reconhecer a pertinência das críticas à saúde atribuindo-as a processos determinados pelos imperativos sistêmicos - econômicos e políticos - sem, contudo, perdê-la como um direito pertencente a todos, relacionado a necessidades dos seres humanos, sendo assim por eles reconhecidos 14,23.

\section{Metodologia}

Foi utilizada associação de vários procedimentos metodológicos (Tabela 1).

\section{Entrevistas semi-estruturadas}

Entrevistas semi-estruturadas, com secretários municipais de saúde, coordenadores de atenção básica e do PSF, supervisores de atenção básica e um representante do Conselho Municipal de Saúde (CMS).

\section{Entrevistas em forma de miniconvenções}

Entrevistas, em forma de miniconvenções, com as equipes de planejamento e controle e avaliação dos municípios envolvidos e com integrantes do Pólo de Educação Permanente.

\section{Entrevistas com profissionais de saúde}

Foram feitas 193 entrevistas com profissionais de saúde - 118 de nível superior e 75 de nível médio - de uma amostra representativa de unidades básicas de saúde locais, escolhidas segundo o Modelo de Atenção à Saúde (unidades com e sem PSF), distribuição geográfica (zonas urbana e rural) e áreas de diferentes perfis de vulnerabilidade, englobando um número que correspondesse no mínimo a $20 \%$ das unidades de saúde de atenção básica em funcionamento no município. 
Procedimentos metodológicos, sujeitos entrevistados e categorias correspondentes.

\begin{tabular}{|c|c|c|}
\hline Procedimentos & Sujeitos da pesquisa & Categorias \\
\hline \multirow[t]{3}{*}{ Entrevistas com informantes-chave } & Secretário Municipal de Saúde; & Funções da Atenção Básica; debate público \\
\hline & Coordenador da Atenção Básica e do PSF; & \\
\hline & Representante do CMS & \\
\hline Miniconvenções & Equipes de saúde da família & Funções da Atenção Básica \\
\hline \multirow[t]{2}{*}{ Entrevistas com profissionais } & Profissionais de nível médio e de nível superior & Relações profissionais/usuários; \\
\hline & das unidades selecionadas & relações profissionais/profissionais \\
\hline Entrevistas semi-estruturadas com usuários & Usuários das unidades selecionadas & Relações profissionais/usuários \\
\hline Entrevistas abertas com usuários & Usuários das unidades selecionadas & Relações profissionais/usuários \\
\hline \multirow[t]{2}{*}{ Grupos focais } & Gerentes e gestores; equipes de avaliação & Mecanismos de controle sistêmicos; \\
\hline & & debate público; relações entre profissionais \\
\hline
\end{tabular}

PSF: Programa Saúde da Família; CMS: Conselho Municipal da Saúde.

\section{Entrevistas com usuários}

a) Entrevistas estruturadas com 411 usuários, segundo amostra do tipo survey para o conjunto dos municípios, calculada com um intervalo de 95\% de confiança e um erro amostral de $5 \%$.

b) Entrevistas abertas orientadas com trinta usuários, escolhidos aleatoriamente em unidades de saúde e unidades com PSF, segundo critério de saturação.

Utilizaram-se os instrumentos adaptados e validados por James Macinko (New York University, New York, Estados Unidos) e Celia Almeida (Fundação Oswaldo Cruz, Rio de Janeiro, Brasil), para entrevistas semi-estruturadas com profissionais e usuários. Os demais instrumentos foram elaborados pelos autores.

\section{Grupos focais}

Foram realizados três grupos focais: (a) com gestores e gerentes dos municípios e do Estado de Mato Grosso sobre a organização da Atenção Básica em cada um; (b) com supervisores de saúde dos municípios e do estado sobre avaliação em saúde e; (c) com gerentes e supervisores do estado e dos municípios sobre as políticas de participação e controle social.

Foi feita análise de implantação pela qual se avalia a relação entre a intervenção de saúde proposta, no caso, a Atenção Básica de Saúde, o contexto de implementação e os efeitos produzidos $24,25,26$.

\section{Procedimentos utilizados na análise dos resultados}

A análise foi desenvolvida em duas partes. Na primeira, foram avaliadas as relações estabelecidas no setor de saúde dos municípios, segundo categorias analíticas definidas pela associação da teoria adotada com os achados empíricos. Levando-se em consideração que o conceito de autonomia implica falar das histórias de vida dos atores, da sua expressão numa rede abstrata de comunicações, e por fim, da capacidade de fazer adentrar no estado, em seus diferentes níveis e setores, os problemas e necessidades da sociedade 12,13, propusemos três categorias de análise: (1) interações entre profissionais/usuários; (2) interações entre os profissionais; e (3) o debate público sobre a saúde e a prática das instâncias institucionalizadas de controle social da saúde, todas três relacionadas ao Mundo da Vida dos participantes. Mas a sociedade, como se viu, é também sistema e daí surge uma quarta categoria de análise, a influência dos mecanismos sistêmicos sobre a saúde. Cada categoria de análise e suas variáveis receberam uma pontuação, atribuída da seguinte maneira: aspectos qualitativos positivos receberam 0,1 ou 2 pontos, segundo referência feita, respectivamente, por nenhum, um, ou mais de um sujeito entrevistado; quanto aos aspectos quantitativos, variáveis com $60 \%$ ou mais, 40 a $60 \%$ e abaixo de $40 \%$ de respostas afirmativas positivas receberam, respectivamente, 2, 1 ou 0 pontos. Referências negativas produzem perda de pontos, 2,1 ou 0 , 
segundo os mesmos critérios. Cada variável recebeu no máximo 2 pontos.

Na segunda parte, a Atenção Básica é analisada segundo suas funções, isto é, atenção ao primeiro contato, coordenação, longitudinalidade e integralidade, utilizando-se o modelo proposto por Starfield 4 . Finalmente, compara-se o desempenho dos municípios segundo a pontuação obtida na primeira e na segunda partes, relacionando assim o tipo das relações estabelecidas e a organização da Atenção Básica.

\section{Resultados e discussão}

\section{Primeira parte: as relações estabelecidas no setor saúde}

\section{- Relações entre usuários e profissionais}

É reconhecido na literatura que a produção da saúde demanda o estabelecimento de relações de intersubjetividade entre profissionais e usuários, abrindo-se a possibilidade de aproximação ao mundo da vida dos últimos, portanto, do seu modo de viver, adoecer e morrer 23,27,28. Relações desse tipo configurariam a ação comunicativa, na qual, os atores envolvidos agem de modo cooperativo para alcançar seus objetivos, orientados por uma expectativa recíproca de reconhecimento de cada um como sujeito portador de competências 12,15. Mas, nos encontros e desencontros de usuários e profissionais dentro do setor saúde 29 nem sempre as coisas se passam assim. Profissionais de saúde podem adotar essa perspectiva que é a dos usuários no seu mundo da vida ou a perspectiva sistêmica, em que esses são receptores passivos de benefícios: sujeitos capazes e responsáveis, no primeiro caso, ou clientes no outro 21,22.

Nos três municípios há preocupação com a avaliação do cuidado pelo usuário, pelo menos no que se refere às reclamações e denúncias que possam comprometer o sistema, todos tendo desenvolvido algum mecanismo de escuta do usuário, especialmente ouvidoria, o que ainda não é suficiente para configurar a primeira perspectiva. É em Rondonópolis que ela surge, a começar pelo desenvolvimento de tecnologia inovadora - a farmácia de manipulação - cuja viabilização contou com a participação de técnicos, poder público municipal, população e CMS. A farmácia é responsável pela fabricação e distribuição de 83 medicamentos. O processo de produção conta com seis farmacêuticos e o controle de qualidade é rigoroso. As prescrições são atendidas em, no máximo, 48 horas; as classificadas como urgentes são aviadas no mesmo dia; há um constante diálogo entre os responsáveis pela produção farmacêutica, os profissionais de saúde e os usuários, propiciando permanente avaliação de mão dupla que subsidia os processos de produção e utilização dos medicamentos. O funcionamento dessa farmácia lembra a proposição de Habermas 22 segundo a qual o Mundo da Vida pode e deve demarcar suas fronteiras com o sistema, fazer prevalecer suas necessidades e não se deixar colonizar. Enumera-se ainda nesse sentido, o desenvolvimento, em Rondonópolis, de práticas de acolhimento e de humanização do cuidado: criação de grupos de trabalho de humanização nas unidades de saúde, sensibilização das equipes sobre o tema, ações de incentivo e valorização do trabalho em equipe e eliminação de intervenções desnecessárias. Na avaliação dos entrevistados, essa é ainda uma prática pouco eficiente e precisa ser melhorada. Em Cuiabá, planeja-se iniciar trabalhos afins e em Várzea Grande a humanização não se mostra como uma prioridade da gestão.

Ponto problemático em Cuiabá e Rondonópolis é a rotatividade dos profissionais, especialmente o médico. O mecanismo utilizado para superar esse problema é a capacitação do profissional, o que acaba sendo um investimento sem retorno para o município. No entanto, todos vêem positivamente esse processo, pois o profissional capacitado, onde estiver, estará contribuindo para a construção do SUS. Rondonópolis e Cuiabá procuram criar formas de incentivo para os profissionais na forma de inserções atrativas em vários espaços do setor. Rondonópolis acrescenta: carreira, organização do trabalho em equipe - que sabidamente, melhora a satisfação dos profissionais 30 - e organização de equipe de supervisão. Várzea Grande não teria o problema da rotatividade, o que se explicaria pelo fato de serem o SUS e a Atenção Básica pouco desenvolvidos neste município, ocupando apenas um tempo residual dos profissionais. Essa explicação se contradiz, pelo menos em parte, pela observação in loco do trabalho de três equipes de saúde da família, atuando em tempo integral, satisfeitos, dedicados. No entanto, o que parece contradição pode ser apenas resultado da ação comunicativa e do compartilhamento do mundo da vida criando coesão, pertencimento e identidade.

A análise dos questionários estruturados mostrou algumas diferenças estatisticamente significativas entre Cuiabá e Rondonópolis. Entre Rondonópolis e Várzea Grande elas são numerosas e entre Cuiabá e Várzea Grande quase inexistentes (Tabela 2). Há de se considerar que os questionários estruturados e analisados segundo a ótica quantitativa não são a melhor maneira 
Avaliação da Atenção Básica por usuários e profissionais de Cuiabá, Várzea Grande e Rondonópolis, Estado de Mato Grosso, Brasil.

\begin{tabular}{|c|c|c|c|}
\hline & Cuiabá & Várzea Grande & Rondonópolis \\
\hline \multicolumn{4}{|l|}{ Avaliação da Atenção Básica por usuários } \\
\hline É atendido pelo mesmo profissional & 81,8 & 61 & 85,2 \\
\hline Fala com o mesmo profissional em caso de dúvida & 63,6 * & 50,0 & 45,7 * \\
\hline O tempo de consulta é suficiente & 78,1 & 53,5 ** & 86,4 ** \\
\hline Usuário entende bem o conselho dado & 85,4 & 55,6 ** & 89,9 ** \\
\hline Usuário tratado como ser humano & 82,2 & $46,0 * \star$ & $87,5 * \star$ \\
\hline Os profissionais conhecem sua família & 29,5 & 15,7 & 23,7 \\
\hline Usuário é ouvido pelo pessoal da unidade de saúde & 21,1 & $14,1 \star \star$ & 32,6 ** \\
\hline Freqüência da visitas domiciliares & 50,5 * & 23,1 ** & $75,0 *$ *** \\
\hline Unidade trabalha com outros grupos & 18,4 & 16,9 & 11,2 \\
\hline O usuário recomenda a unidade de saúde a um amigo & 89,8 * & 63,4 & 72,7 * \\
\hline Profissionais têm boa relação com a comunidade & 87,7 & 69,8 ** & $92,9 * \star$ \\
\hline Os profissionais conhecem os problemas mais importantes da comunidade & 62,5 & 42,3 ** & 58,4 ** \\
\hline As autoridades pedem a representantes da comunidade para participarem da direção & 15,3 * & 4,2 ** & $41,6 *, * \star$ \\
\hline A unidade oferece serviço às escolas & 13,3 & 16,7 ** & $20,2 * \star$ \\
\hline \multicolumn{4}{|l|}{ Avaliação da Atenção Básica por profissionais } \\
\hline Os pacientes são avaliados pelo mesmo profissional & 92,6 * & 95,2 & 80,6 * \\
\hline Profissional responde a dúvidas do paciente & 56,7 & 66,7 & 64,0 \\
\hline Tempo de consulta é adequado & 89,9 & 76,2 & 87,2 \\
\hline População adscrita & 70,3 & 70,0 & 71,6 \\
\hline Realiza enquetes para avaliar serviço & $50,0 * \star \star$ & $23,8 * \star \star$ & 39,4 \\
\hline Realiza enquetes para identificar problema & 47,8 & 42,9 & 41,5 \\
\hline Tem representantes da unidade participando da direção da unidade & 58,2 * & $38,1 * \star$ & $76,6 * * *$ \\
\hline Realiza trabalhos intersetoriais & 41,2 & 47,6 & 48,9 \\
\hline Equipe foi capacitada & 60,9 & 57,1 & 60,9 \\
\hline Anamnese inclui pergunta sobre a família & 90,8 & 88,9 & 83,5 \\
\hline
\end{tabular}

* Diferenças significativas entre Cuiabá e Rondonópolis;

** Diferenças significativas entre Várzea Grande e Rondonópolis;

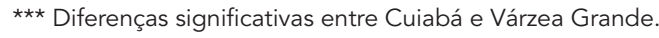

para comparar aspectos qualitativos, pelo menos quando eles não são radicalmente diferentes. Além disso, a pesquisa com usuários foi realizada dentro das unidades de saúde e sabe-se que haveria diferença importante caso fossem realizadas longe do serviço 31 , o que pode ser atribuído à satisfação momentânea com o atendimento que acabara de ocorrer ou ao receio, fundado ou não, de retaliações.

Merece maiores investigações o fato de ser alta a satisfação dos usuários em todos os três municípios, apesar das diferenças de organização do setor entre eles. Uma explicação para o fato seria a de que relações simétricas e participativas contribuam também para o desenvolvimento da capacidade crítica e reivindicativa dos atores sociais, o que é compatível com o modelo de democracia proposto por Habermas 12,13,21,22.

\section{- Relações entre os profissionais: equipe}

O trabalho em equipe desempenha papel fundamental na Atenção Básica de Saúde - equipes coesas se associam com melhores resultados e maior satisfação dos pacientes e dos profissionais 4,31 . Contudo, não basta que os profissionais estejam no mesmo espaço físico - é preciso que tenham competência complementar, com papéis definidos, desenvolvendo trabalho compartilhado e cooperativo 4,32 . Logo, equipe é uma rede de relações, permeada por múltiplos elementos - competências, afetos, poderes 33 . Representa um dos elementos-chave dos novos modelos de atenção 33,34, e, paradoxalmente, é um dos maiores desafios a ser enfrentado, dadas as barreiras impostas pelas disciplinas e os desafios das relações e personalidades humanas 4,31, muito competitivas no nosso tempo. Habermas 
fala de colonização do Mundo da Vida quando as interações mediadas pelo entendimento lingüístico são substituídas por imperativos sistêmicos, processo que marca fortemente as sociedades modernas, globalizadas e complexas 15,22: é sabido como estas características dificultam ou impedem a intersubjetividade e o trabalho solidário.

Em todos os três municípios, as equipes são multidisciplinares e formadas segundo o padrão básico - médico, enfermeiro, auxiliar de enfermagem e agentes comunitários. Têm atribuições específicas e bem definidas - o problema fica por conta da complementaridade e da cooperação. Sabe-se que a supervisão pode melhorar esse desempenho 34 . Rondonópolis, mas não os outros dois municípios, dispõe de uma equipe de supervisão integrada, com objetivos, atribuições e métodos de trabalho compartilhados e mecanismos bem definidos de comunicação entre os integrantes. Essa equipe se encarrega da supervisão/avaliação de todas as unidades do sistema, PSF ou não, ao contrário dos outros dois municípios e do próprio estado que têm profissionais com a tarefa de supervisionar apenas as unidades do PSF (Tabela 3).

\section{- O debate público e os processos institucionalizados de participação}

Na teoria habermasiana, o estabelecimento de relações simétricas se associa, de forma circular, com o estabelecimento de mecanismos democráticos. O mesmo ocorre com a atenção de saúde 35,36,37,38,39. Em nenhum dos municípios há um movimento social voltado para a saúde, duradouramente constituído. No entanto, é possível notar diferenciações. Os atores entrevistados de Rondonópolis tematizam espontaneamente esse elemento e agem tentando efetivá-lo. Se levarmos em consideração a análise de Saltmann 33, segundo a qual, pessoas participam quando são ouvidas e chamadas a participar, há um dado significativo da fala dos usuários - quando perguntados sobre convite para participar, os entrevistados de Rondonópolis dizem que sempre são chamados pelas autoridades, situação oposta à dos outros dois, onde a quase totalidade responde que nunca é chamada.

Quanto às instâncias institucionalizadas de controle social do setor saúde, são as que, por excelência, deveriam garantir a participação nas decisões e possibilitar que a população tenha voz e influência para fazer valer seus interesses frente aos outros interesses que permeiam o setor. A sua ampla existência e sua composição paritária nem sempre garantem essa participação, mais uma vez, entrando em pauta as relações que aí se travam. São conhecidos os argumentos que apontam para o predomínio técno-burocrático das decisões dentro dos CMSs 40,41. Fazendo analogia com o modelo de democracia proposto por Habermas, as instâncias institucionalizadas só efetivarão o controle social sobre o sistema de saúde se se vincularem ao debate público. Nos três municípios, existem mecanismos de divulgação das decisões do CMS - jornal, boletim, ou rádio - o que é promissor, pois não há qualquer debate se as informações não fluem livremente 12 . Mas há diferenças nos municípios quanto à influência do CMS, sugeridas pela participação dos profissionais e do Secretário nas reuniões e pela presença efetiva na rotina do setor de saúde; à realização de conferências, de reuniões comunitárias e de debate público. Rondonópolis conta com Comissões Locais de Saúde, institucionalizadas, em todas as unidades, muitas delas sob a presidência de um usuário. Essas comissões são estimuladas a manter aberto o debate sobre saúde e a subsidiar as decisões do CMS e das Conferências. Há um esforço grande da gestão para efetivar as diretrizes apontadas nessas instâncias. As prioridades do Plano de Saúde e do Relatório de Gestão são as aprovadas nas Conferências. O fortalecimento do Controle Social é a prioridade número 1 do município (Tabela 3 ).

\section{- A influência dos mecanismos sistêmicos de controle: dinheiro e poder}

Nas falas dos entrevistados há referências à influência política, que se expressa no recrutamento dos quadros da saúde e na definição das prioridades, do investimento, do modelo de atenção. Do mesmo modo, há referência aos interesses econômico-financeiros, expressos na rotatividade, tipo de inserção dos profissionais de saúde, em todos os seus níveis de atuação e, sobretudo, nos compromissos prioritários estabelecidos com o setor público ou com o setor privado de saúde. Segundo nossa abordagem teórica, elementos políticos e econômicos têm um papel óbvio para a sociedade. Coisa diferente é falar da preponderância desses elementos sobre os interesses gerais da sociedade. Repete-se aqui a tese da colonização do mundo da vida que só nas falas coletivas - grupos focais, oficinas, reuniões - se configura com toda a clareza - fala-se, e há um consenso sobre isso, dos "feudos e famílias poderosas", da "sangria" de recursos públicos e do "controle político das práticas de saúde", situações também diferenciadas para os três municípios (Tabela 3). 
Pontuação obtida pelos municípios de Cuiabá, Rondonópolis e Várzea Grande, Estado de Mato Grosso, Brasil, referentes às relações estabelecidas dentro do sistema de saúde.

\begin{tabular}{|c|c|c|c|}
\hline Categorias e critérios & Cuiabá & Rondonópolis & Várzea Grande \\
\hline \multicolumn{4}{|l|}{ Trabalho em equipe } \\
\hline 1. Objetivos bem definidos & - & 2 & 1 \\
\hline 2. Atribuições bem definidas & 1 & 2 & 1 \\
\hline 3. Planejamento participativo & - & 2 & - \\
\hline 4. Mecanismos de comunicação & 1 & 2 & - \\
\hline 5. Política de recursos humanos & 1 & 2 & 1 \\
\hline 6. Autonomia & 2 & 2 & - \\
\hline 7. Perfil inadequado* & -2 & -2 & -2 \\
\hline 8. Alta rotatividade * & -2 & -2 & - \\
\hline \multicolumn{4}{|l|}{ Relação usuário/equipe } \\
\hline 1. Acolhimento & - & 1 & - \\
\hline 2. Humanização & 1 & 1 & - \\
\hline 3. Compromisso explícito com o usuário & - & 2 & - \\
\hline 4. Desenvolvimento de tecnologias inovadoras & - & 2 & - \\
\hline 5. Comunicação profissional/usuário & 2 & 2 & - \\
\hline 6. Avaliação do cuidado pelo usuário - ouvidoria & 2 & 2 & 2 \\
\hline 7. Avaliação do serviço pelo usuário - caixa de reclamações & 2 & - & 2 \\
\hline 8. Avaliação do serviço pelo usuário - pesquisa & 1 & - & - \\
\hline 9. Avaliação do serviço pelo usuário - Disque Denúncia & - & 1 & 1 \\
\hline 10. Realização de enquetes: o serviço responde às necessidades? & 1 & - & - \\
\hline 11. Participação na organização do serviço & 2 & 2 & - \\
\hline 12. Criação de espaços e mecanismos de participação & 2 & 2 & 1 \\
\hline 13. Conhecimento pela equipe dos problemas dos usuários da sua área & 2 & 1 & 1 \\
\hline 14. Mecanismos de informação & 2 & 1 & - \\
\hline 15. Organização de atividades intersetoriais junto com a comunidade & - & 2 & - \\
\hline 16. Satisfação do usuário & 2 & 2 & 1 \\
\hline 17. Atendimento inadequado, desumanizado * & -2 & - & -1 \\
\hline \multicolumn{4}{|l|}{ Processos de participação e ênfase ao desenvolvimento de cidadania } \\
\hline 1. Convite à participação & - & 2 & - \\
\hline 2. Compromisso explícito com direito de saúde de todo cidadão & - & 2 & - \\
\hline 3. Compromisso explícito com a participação do usuário e da população & - & 2 & - \\
\hline como mecanismo de produção da saúde com qualidade & - & - & - \\
\hline 4. Adoção de propostas amplamente debatidas & - & 2 & - \\
\hline 5. Prestígio do conselho & - & 2 & 1 \\
\hline 6. Influência nas decisões & 1 & 2 & - \\
\hline 7. Participação dos profissionais & 1 & 1 & - \\
\hline 8. Formulação de políticas & 1 & 1 & - \\
\hline 9. Mecanismos de incorporação das necessidades da população & 2 & 2 & - \\
\hline 10. Mecanismos de informação e divulgação & 1 & 2 & - \\
\hline 11. Comissões locais & 2 & 2 & - \\
\hline 12. Reuniões comunitárias & - & 1 & - \\
\hline 13. Debate público & - & 1 & - \\
\hline 14. Mecanismos que ampliam a participação & 2 & 1 & - \\
\hline 15. Críticas à interferência política & 2 & 2 & 2 \\
\hline 16. Preponderância dos interesses políticos * & -2 & - & -2 \\
\hline 17. Preponderância dos interesses econômicos e financeiros * & -2 & - & -2 \\
\hline Total & 28 & 43 & 7 \\
\hline
\end{tabular}

* Critérios negativos que acarretam perda de pontos, quando existentes. 
Segunda parte: a análise da Atenção Básica segundo suas funções

A Atenção Básica não deve ser entendida como um dos diferentes espaços de prestação de cuidado individual do sistema de saúde, pois esta é uma perspectiva reduzida em relação ao papel que ela desempenha na integração, efetividade e eficiência do sistema como um todo. Ela melhora o funcionamento do serviço de saúde e a qualidade do cuidado de várias formas 4,42: gerenciando os casos indiferenciados; combinando as necessidades dos pacientes com os cuidados prestados; otimizando os recursos, evitando faltas ou excessos no tratamento; aumentando a capacidade de adaptação do sistema a novas circunstâncias e reduzindo as disparidades geográficas e sócio-econômicas.

Em relação ao usuário e à população, a Atenção Básica ainda propicia: acompanhamento e cuidado duradouros de pacientes com problemas indiferenciados; conhecimento do usuário, das famílias e da comunidade, permitindo ao profissional antecipar/ajustar cuidados a serem adotados; melhor relação usuário/população/ sistema de saúde, com estabelecimento de laços de confiança; melhor acesso ao cuidado de saúde nesse nível de atenção; papel de ponte entre a comunidade e os serviços de saúde de diferente complexidade; maior probabilidade de atuar em regiões mais carentes.

Starfield ${ }^{4}$ define como funções essenciais da Atenção Básica: (1) atenção ao primeiro contato, (2) integralidade, (3) coordenação e (4) longitudinalidade. Essa última função, segundo a autora, exige o estabelecimento de fortes relações intersubjetivas que "reflitam a cooperação mútua entre as pessoas e os profissionais de saúde" 4 (p. 61 ), logo se confunde com as interações tomadas como referência neste trabalho e dispensa comentários adicionais.

A garantia de atenção ao primeiro contato significa que a Atenção Básica é porta de entrada das questões de saúde, lida com problemas amplos, heterogêneos e fortemente influenciados pelo contexto social. A integralidade implica o caráter inclusivo dos serviços oferecidos de modo a permitir que o usuário receba todos os tipos de cuidados necessários, desde a promoção da qualidade de vida e prevenção de riscos, até a oferta em todos os níveis - primário, secundário e terciário - de cuidados de recuperação e reabilitação. A coordenação implica garantir a continuidade e o acompanhamento de saúde da população e de cada usuário de um modo integrado e organizado, o que por sua vez exige a disponibilidade das informações ao longo do tempo e em diferentes espaços 4 .
As categorias propostas por Starfield ${ }^{4}$ para avaliação dessas funções são: (1) atenção ao primeiro contato - acessibilidade e utilização do serviço pelo usuário; (2) longitudinalidade - adscrição da população, continuidade e vínculo; (3) integralidade - variedade dos serviços e capacidade de diagnóstico, reconhecimento e manejo dos problemas; (4) coordenação - continuidade e reconhecimento, diagnóstico e abordagem dos problemas. Neste trabalho, utilizaremos essas mesmas categorias (Tabela 4).

A acessibilidade é parâmetro de avaliação das barreiras que se interpõem ao acesso ao cuidado de saúde, que podem ser de ordem geográfica, econômica, organizacional e cultural 4,43.

A análise qualitativa mostrou que há uma tensão entre Cuiabá e Várzea Grande, em razão da proximidade de ambos e da sobrecarga de demanda do primeiro, originária de usuários do segundo, onde a oferta de serviços é menor do que as necessidades da população. Por seu turno, Cuiabá ainda não consolidou o modelo de atenção regionalizado e hierarquizado, tenta se recuperar de uma crise do setor saúde e, no momento, a alternativa adotada é o início de estruturação da Rede de Atenção Básica. Em Rondonópolis há cobertura universal de Atenção Básica pelo setor público, sendo o modelo consolidado, com 29 equipes de PSF garantindo $60 \%$ da cobertura. Nos três municípios, o tempo de espera para obtenção de consulta gira em torno de dois a sete dias. Não se detectou, em nenhum município o co-pagamento de consultas. A oferta de cuidados básicos, incluindo atendimento e medicamentos, é garantida em Rondonópolis para toda a população, mas há problemas com o acesso a outros níveis de complexidade. Em Cuiabá, ainda há dificuldades de acesso em todos os níveis de atenção, em compensação, há um programa de saúde bucal bem desenvolvido e organizado e também uma iniciativa incipiente de produção e uso de fitoterápicos.

A utilização do serviço tem a ver com a percepção do usuário sobre a disponibilidade e a qualidade do serviço e se aproxima da aceitabilidade e da legitimidade a ele conferidas pelo usuário, dois pilares da qualidade propostos por Donabedian 44 . As entrevistas semi-estruturadas com usuários mostram que $71 \%$ dos de Cuiabá consideram fácil conseguir uma consulta sempre ou quase sempre e $51 \%$ deles responderam que têm sempre ou quase sempre à sua disposição os medicamentos e equipamentos necessários, marcas bem melhores do que as de Rondonópolis, com $58 \%$ e $21 \%$, e Várzea Grande, com 53\% e $11 \%$, respectivamente.

A função de integralidade relaciona-se, influencia e, às vezes, até mesmo se confunde com 
Análise da Atenção Básica dos municípios de Cuiabá, Rondonópolis e Várzea Grande, segundo as funções propostas por Starfield 4 e respectivas pontuações.

\begin{tabular}{|c|c|c|c|}
\hline Categorias e critérios & Cuiabá & Rondonópolis & Vargem Grande \\
\hline \multicolumn{4}{|l|}{ 1. Atenção ao primeiro contato } \\
\hline Acessibilidade & - & - & - \\
\hline Adoção do modelo de atenção de saúde com ênfase para Atenção Básica & - & 2 & - \\
\hline Oferta de serviços, equipamentos e medicamentos & 1 & 1 & - \\
\hline Co-pagamento de consultas & 2 & 2 & 2 \\
\hline Prazo de obtenção de consulta & 2 & 2 & 2 \\
\hline Existência de telefone de agendamento & - & 1 & - \\
\hline Desenvolvimento de tecnologias inovadoras & - & 2 & - \\
\hline Utilização do serviço & - & - & - \\
\hline Facilidade para conseguir consultas & 2 & 1 & 1 \\
\hline Oferta de medicamentos e equipamentos & 1 & - & - \\
\hline \multicolumn{4}{|l|}{ 2. Integralidade } \\
\hline Equipes multidisciplinares & 2 & 2 & 2 \\
\hline Variedade de serviços & 2 & 2 & 2 \\
\hline Capacitação de profissionais & 1 & 1 & 1 \\
\hline Preocupação em ampliar o serviço & 2 & 2 & 2 \\
\hline Oferta de serviços da Atenção Básica & 1 & 2 & - \\
\hline Ações voltadas para prevenção e promoção & 0 & 1 & - \\
\hline \multicolumn{4}{|l|}{ 3. Coordenação } \\
\hline Normas e fluxos entre os diferentes níveis de Atenção Básica & 1 & 2 & - \\
\hline Serviços de referência e contra-referência & 1 & 1 & - \\
\hline Existência de protocolos & 1 & 2 & - \\
\hline Instrumentos organizados de avaliação e monitoramento & - & 2 & - \\
\hline Integração da equipe & - & 2 & - \\
\hline Mecanismos de comunicação entre os profissionais & - & 2 & - \\
\hline Total & 19 & 32 & 12 \\
\hline
\end{tabular}

os demais princípios e diretrizes do SUS 28,45,46,47. Em todos os municípios as equipes são multidisciplinares e há uma variedade de serviços disponíveis, como saúde da criança, da mulher, programas do Ministério da Saúde (tuberculose, hanseníase, hipertensão, diabetes, programas de vacinação, saúde do idoso etc.), políticas específicas, como saúde do adolescente etc.; acesso a várias especialidades e diferentes propedêuticas etc. A capacitação de profissionais de saúde também é presente em todos os três municípios, embora ainda seja considerada insuficiente e muitas vezes inadequada. Ainda que o investimento na expansão do serviço esteja presente como preocupação em todos os municípios, é unânime entre os atores entrevistados que em Várzea isso ainda se restringe ao discurso. As ações voltadas para a prevenção e promoção são praticamente ausentes nesse município e isto é atribuído à pequena capacidade organizacional da Secretaria, à carência de recursos e de pessoal. Em Cuiabá, essas ações ficam na dependência da iniciativa de cada equipe. Em Rondonópolis, existe uma preocupação coletiva e esforços são expendidos para o desenvolvimento de práticas de controle de riscos, como alcoolismo, obesidade, drogadição etc. e a participação em programas de geração de renda e melhoria da qualidade de vida da população, mas na avaliação dos entrevistados ainda são programas incipientes.

Por fim, a função de coordenação diz respeito à capacidade integradora e racionalizadora do sistema e ao uso de informações sobre o cuidado individual e coletivo, ao longo do tempo e nos diferentes espaços, subsidiando as decisões e o planejamento do trabalho ${ }^{4}$. A diferenciação de Rondonópolis, em relação aos demais, se faz, especialmente aqui, pelo investimento na organização do serviço, o que se percebe nas falas, nos grupos focais, na observação de campo e nos documentos disponibilizados, com suas normas e fluxos definidos de informação entre os diferentes níveis de atenção, de atuação da Atenção Básica e de referência e contra-referência (cuida- 
dos especializados e propedêutica complementar); com seus protocolos, seus registros e seus instrumentos organizados de avaliação e monitoramento e com suas equipes integradas e mobilizadas. Quando perguntado no grupo focal a que se atribuía diferença tão reiterada, a resposta vem de pronto: "ao perfil e à vontade política, ao empenho e ao compromisso com o setor saúde". Quando alguns atores se queixaram da inexistência de condições políticas favoráveis, novamente a resposta é precisa: "em Rondonópolis também era assim - o que é preciso é começar o processo de mudança".

\section{Conclusão}

O pressuposto deste trabalho é de que as relações entre os diferentes atores são decisivas na organização da Atenção Básica e na mudança do modelo de atenção, e os dados empíricos obtidos são compatíveis com este pressuposto. Em todos os procedimentos é possível apreender que relações de intersubjetividade travadas entre os profissionais entre si e destes com os usuários são fundamentais no desempenho das funções da atenção básica, o que por sua vez, se relaciona com o maior ou menor grau de mobilização da população e de desenvolvimento do debate público. Por isso, foi possível encontrar correspondência entre o nível de desenvolvimento da Atenção Básica e o tipo de relações estabelecidas - os escores obtidos na primeira e na segunda partes, resultantes da pontuação de variáveis quantitativas e qualitativas demonstram esta correspondência. Corroborando esses resultados, constatase um desenvolvimento maior de Rondonópolis, que se manifesta em todas as categorias de análise, principalmente, a organização da equipe, a relação profissional/usuário e o desempenho das funções da atenção básica. É consensual a avaliação de que esse avanço se deva ao tipo de opinião e vontade políticas ali predominantes. A análise estatística dos questionários respondidos pelos usuários e pelos profissionais não conseguiu apreender, por si, a maioria das diferenças, mas não as refuta.

As limitações de cada procedimento utilizado referem-se principalmente às influências de diferentes variáveis sobre a fala dos entrevistados e foram tratadas pela associação de diferentes procedimentos aplicados a sujeitos com papéis, interpretações e preferências diferentes, às vezes polarizadas.

\section{Resumo}

Este trabalho engloba parte dos resultados do Estudo de Linhas de Base, Projeto de Consolidação e Expansão do Saúde da Família (PROESF). Seu objetivo é avaliar a Atenção Básica dos municípios de Cuiabá, Várzea Grande e Rondonópolis, Estado de Mato Grosso, Brasil, o que será feito segundo as relações de intersubjetividade que lhes são próprias - entre os profissionais de saúde, entre estes e os usuários, entre os cidadãos em geral e dentro das instâncias institucionalizadas de controle social. É um estudo quali-quantitativo, composto de entrevistas com informantes-chave, miniconvenções com gerentes, grupos focais com gerentes, entrevistas com usuários e profissionais das unidades de saúde selecionadas. Foram atribuídos escores para os quesitos indicativos de processos participativos e para as funções da Atenção Básica. Constatou-se, apesar da identidade geopolítica dos municípios e do acesso deles às mesmas políticas públicas, diferença significativa quanto ao desempenho das funções relativas à organização da Atenção Básica e do Programa Saúde da Família: porta de entrada, longitudinalidade, integralidade e coordenação. Constataram-se ainda diferenças referentes ao tipo de relações estabelecidas, participativas ou não, em correspondência com a diferença anterior.

Atenção Primária à Saúde; Avaliação; Programa Saúde da Família

\section{Colaboradores}

E. M. Melo escreveu o artigo e participou da construção do desenho da pesquisa e da execução do trabalho de campo. L. Paiva, J. Álvares e A. L. D. Hecha participaram da construção do desenho da pesquisa, da execução do trabalho de campo e da redação da metodologia do artigo. 


\section{Referências}

1. Pinheiro RS, Ecosteguy CC. Epidemiologia e serviços de saúde. In: Medronho RA, organizador. Epidemiologia. São Paulo/Rio de Janeiro/Ribeirão Preto: Editora Atheneu; 2003. p. 261-9.

2. Canguilhem G. O normal e o patológico. 3a Ed. Rio de Janeiro: Editora Forense Universitária; 1990.

3. Mendes EV. Uma agenda para a saúde. São Paulo: Editora Hucitec; 1996.

4. Starfield B. Atenção primária. Equilíbrio entre necessidades de saúde, serviços e tecnologia. Brasília: Organização das Nações Unidas para a Educação, a Ciência e a Cultura; 2002.

5. Goulart FAA. Experiências em saúde da família: cada caso é um caso? [Tese de Doutorado]. Rio de Janeiro: Escola Nacional de Saúde Pública, Fundação Oswaldo Cruz; 2002.

6. Paim J. Bases conceituais da reforma sanitária brasileira. In: Fleury SF, organizador. Saúde e democracia: a luta do CEBES. São Paulo: Editorial Lemos; 1997. p. 11-24.

7. Instituto Brasileiro de Geografia e Estatística. Cidades. http://www.ibge.gov.br/cidades (acessado em 30/Abr/2007).

8. Programa das Nações Unidas para o Desenvolvimento/Instituto de Pesquisa Econômica Aplicada/Fundação João Pinheiro/Instituto Brasileiro de Geografia e Estatística. Atlas de Desenvolvimento Humano no Brasil, 2000. http://www.ibge.gov.br (acessado em 30/Abr/2007).

9. World Health Organization. Primary health care. Geneva: World Health Organization; 1978.

10. Donnangelo MCF. Saúde e sociedade. 2a Ed. São Paulo: Livraria Duas Cidades; 1979.

11. Arouca ASS. O dilema preventivista. Contribuição para compreensão e crítica da medicina preventiva [Tese de Doutorado]. Campinas: Faculdade de Ciências Médicas, Universidade Estadual de Campinas; 1975.

12. Habermas J. Between facts and norms. Contributions to a discourse theory of law and democracy. Cambridge: The MIT Press;1996.

13. Habermas J. A inclusão do outro. Estudos de teoria política. 2a Ed. São Paulo: Edições Loyola; 2004

14. Melo EM. Ação comunicativa, democracia e saúde. Ciênc Saúde Coletiva 2005; 10:167-8.

15. Habermas J. Teoría de la acción comunicativa. Madrid: Editorial Taurus; 1987.

16. Habermas J. Pensamento pós-metafísico. Rio de Janeiro: Editora Tempo Brasileiro; 1990.

17. Habermas J. Teoría de la acción comunicativa: complementos y estudios previos. Madrid: Editorial Cátedra; 1989.

18. Habermas J. Verdad y justificación. Madrid: Trotta; 2002.

19. Habermas J. Técnica e ciência como ideologia. In: Os pensadores. v. LVIII. São Paulo: Abril Cultural; 1975.

20. Habermas J. Legitimation crisis. Boston: Beacon Press; 1975.

21. Habermas J. Un modelo del compromiso del Estado. Teorema 1983; 13:9-10.

22. Habermas J. A nova intransparência. A crise do estado de bem-estar social e esgotamento das energias utópicas. Novos Estudos CEBRAP 1987; 18:103-14.
23. Melo EM. Fundamentos para uma proposta democrática de saúde: a teoria da ação comunicativa de Habermas [Tese de Doutorado]. Ribeirão Preto: Faculdade de Medicina de Ribeirão Preto, Universidade de São Paulo; 1999.

24. Hartz ZMA, Vieira-da-Silva LM. Avaliação em saúde. Dos modelos teóricos à prática na avaliação de programas e sistemas de saúde. Salvador: EdUFBA/ Rio de Janeiro: Editora Fiocruz; 2005.

25. Constandrioupoulos AP, Champagne F, Denis JL, Pineault R. A avaliação na área de saúde: conceitos e métodos. In: Hartz MZA, organizador. Avaliação em saúde. Dos modelos à prática na análise de implantação de programas. Rio de Janeiro: Editora Fiocruz; 1997. p. 29-47.

26. Hartz ZMA, Pourvourville G. Avaliação dos programas de saúde: a eficiência em questão. Ciênc Saúde Coletiva 1998; 3:68-82.

27. Ayres R. Norma e formação: horizontes filosóficos para as práticas de avaliação no contexto da promoção de saúde. Ciênc Saúde Coletiva 2004; 9:583-92.

28. Merhy EE. A perda da dimensão cuidadora na produção de saúde - uma discussão do modelo assistencial e da intervenção no seu modo de trabalhar a assistência. In: Campos CR, Malta DC, Reis AT, Santos AF, Merhy EE, organizadores. Sistema Único de Saúde em Belo Horizonte: reescrevendo o público. São Paulo: Xamã Editora; 1998. p. 103-20.

29. Craco PF. Ação comunicativa no cuidado da saúde da família: encontros e desencontros entre profissionais de saúde e usuários [Tese de Doutorado]. Ribeirão Preto: Escola de Enfermagem de Ribeirão Preto, Universidade de São Paulo; 2006.

30. Grumbach K, Bodenheimer T. Can health care teams improve primary care practice? JAMA 2004; 291:1246-51.

31. Bosi ML, Mercado FJ. Pesquisa qualitativa de serviços de saúde. Petrópolis: Editora Vozes; 2004.

32. Organização Pan-Americana da Saúde/Núcleo de Estudo em Saúde Coletiva, Universidade Federal de Minas Gerais. Organização do cuidado a partir de problemas: uma alternativa metodológica para atuação da equipe de saúde da família. Belo Horizonte: Organização Pan-Americana da Saúde/Núcleo de Estudo em Saúde Coletiva, Universidade Federal de Minas Gerais; 2000.

33. Fortuna CM, Mishima SM, Matumoto S, Pereira MJB. O trabalho de equipe no programa de saúde da família: reflexões a partir de conceitos do processo grupal e de grupos operativos. Rev Latinoam Enfermagem 2005; 13:262-8.

34. Ministério da Saúde. Programa Saúde da Família. Brasília: Ministério da Saúde; 1996.

35. Saltmann RB. Patient empowerment in Northern European health systems: a conceptual framework. Int J Health Serv 1994; 24:201-29.

36. Campos GWS. A saúde pública e a defesa da vida. 2a Ed. São Paulo: Editora Hucitec; 1994.

37. Andrade GRB, Vaitsman J. Apoio social e redes: conectando solidariedade e saúde. Ciênc Saúde Coletiva 2002; 7:925-34. 
38. Gerschmann S. A democracia inconclusa: um estudo da reforma sanitária brasileira. 2a Ed. Rio de Janeiro: Editora Fiocruz; 2004.

39. Sperandio AMG, Correa CRS, Serrano MM, Rangel HU. Caminho para a construção coletiva de ambientes saudáveis. Ciênc Saúde Coletiva 2004; 9:643-54.

40. Ribeiro JM. Conselhos de saúde, comissões intergestores e grupos de interesses no Sistema Único de Saúde (SUS). Cad Saúde Pública 1997; 13:81-92.

41. Wendhausen A, Caponi S. O diálogo e a participação em um conselho de saúde em Santa Catarina, Brasil. Cad Saúde Pública 2002; 18:1621-8.

42. Ferrer RL, Hambidge SJ, Maly RC. The essential role of generalists in health care systems. Ann Intern Med 2005; 142:691-9.

43. Lopes RM, Vieira-da-Silva LM, Hartz ZMA. Teste de metodologia para avaliar a organização, acesso e qualidade técnica do cuidado na atenção à diarréia na infância. Cad Saúde Pública 2004; 20 Suppl 2:S283-97.
44. Donabedian A. The seven pillars of quality. Arch Pathol Lab Med 1990; 114:1115-8.

45. Conill E. Avaliação da integralidade: conferindo sentido para os pactos na programação de metas dos sistemas municipais de saúde. Cad Saúde Pública $2004 ; 20: 1417-23$.

46. Silva Júnior AG, Mascarenhas MTM. Avaliação da atenção básica em saúde sob a ótica da integralidade: aspectos conceituais e metodológicos. In: Pinheiro R, Mattos RA, organizadores. Cuidado: as fronteiras da integralidade. São Paulo: Editora Hucitec/Rio de Janeiro: Instituto de Medicina Social, Universidade do Estado do Rio de Janeiro/ABRASCO, 2004. p. 241-57.

47. Mattos RA. A integralidade na prática (ou sobre a prática da integralidade). Cad Saúde Pública 2004; 20:1411-6.

Recebido em 22/Fev/2007

Versão final reapresentada em 07/Mar/2007

Aprovado em 09/Mai/2007 